\title{
Visual Outcomes with Flow-Diverter Stents Covering the Ophthalmic Artery for Treatment of Internal Carotid Artery Aneurysms
}

\author{
A. Rouchaud, O. Leclerc, Y. Benayoun, S. Saleme, Y. Camilleri, F. D'Argento, M.-P. Boncoeur, P.-Y. Robert, and C. Mounayer
}

\begin{abstract}
BACKGROUND AND PURPOSE: Flow-diverting stents can be used to treat intracranial aneurysms that are not amenable to treatment with coils. We analyzed ophthalmic consequences due to coverage of the origin of the ophthalmic artery by flow-diverting stents for the treatment of internal carotid artery aneurysms.
\end{abstract}

MATERIALS AND METHODS: From April 2009 to April 2013, the clinical and angiographic outcomes of all 28 patients treated for aneurysms with flow-diverting stents covering the origin of the ophthalmic artery were prospectively collected. The origin of the ophthalmic artery in relation to the target aneurysm was classified by using a 4-type classification. A complete ophthalmic examination was performed by a single ophthalmologist 48 hours before and 1 week after covering the ophthalmic artery.

RESULTS: Ophthalmic artery patency was normal at the end of endovascular treatment in $24 / 28$ cases (85.7\%). With extensive ophthalmic examinations, 11 patients (39.3\%) showed new ophthalmic complications. Patients with the ophthalmic artery originating from the aneurysm sac were at high risk for retinal emboli $(4 / 5,80 \%)$. Patients with the ophthalmic artery originating from the inner curve of the carotid siphon were at high risk for optic nerve ischemic atrophy (3/4, 75\%).

CONCLUSIONS: This prospective study shows that covering the ophthalmic artery with a flow-diverting stent is not without potential complications. Ophthalmic complications can occur but are often not diagnosed. The anatomic disposition of the ophthalmic artery in relation to the carotid siphon and aneurysm should be clearly understood because some configurations have a higher risk. When not required, covering of the ophthalmic artery by flow-diverting stents should be avoided.

ABBREVIATIONS: FDS = flow-diverting stent; $\mathrm{OA}^{2}=$ ophthalmic artery aneurysm; $\mathrm{PED}=$ Pipeline Embolization Device

$F$ ow-diverting stents (FDSs) are commonly used for the endovascular reconstruction of a segmentally diseased parent vessel and treatment of large-neck intracranial aneurysms, which can be difficult to reconstruct with coils, even when used with large-cell stents. ${ }^{1-6}$ FDSs aim to maintain normal blood flow through parent and branch vessels while disrupting flow into the aneurysm, causing thrombosis, and eventually sealing the aneurysm ostium through neointimal proliferation across the device struts. ${ }^{6-8}$ Good angiographic and clinical results have been achieved in small series. ${ }^{4-6,9-12}$

One issue concerning intracranial aneurysm treatment by an FDS is the patency of the perforating arteries and side branches

Received April 14, 2014; accepted after revision August 14.

From the Departments of Interventional Neuroradiology (A.R., S.S., Y.C., M.-P.B., C.M.) and Ophthalmology (O.L., Y.B., P.-Y.R.), Centre Hospitalier et Universitaire Dupuytren, Limoges, France; and Institute of Radiology (F.D.), Policlinico Agostino Gemelli, Catholic University, Rome, Italy.

Please address correspondence to Aymeric Rouchaud, MD, Department of Interventional Neuroradiology, CHU Dupuytren, 2 Ave Martin Luther King, Limoges, France; e-mail: aymeric.rouchaud@gmail.com

http://dx.doi.org/10.3174/ajnr.A4129 covered by the device. ${ }^{13}$ FDSs are designed to provide sufficient coverage across the aneurysm neck to exclude the lesion from circulation but to be porous enough to preserve the patency of any branch vessels covered by the construct through the interstices between the device strands. ${ }^{13}$ The flow into the regional perforators and branch arteries is governed by different factors from those that drive flow into an aneurysm, and the patency of the perforators is maintained by a suction effect due to lower pressure in those branches. ${ }^{14}$ This has been studied with histologic evaluation of the Pipeline Embolization Device (PED; Covidien, Irvine, California) in rabbit aorta, demonstrating rounded funnel-like defects into the homogeneous sheet of neoendothelium on the FDS in regard to the orifices of the regional branches. ${ }^{15}$

This pressure gradient may, however, be less important in vessels that have robust competitive collateral flow. ${ }^{15}$ This is most frequently seen when constructs are built over the ophthalmic artery, which occasionally becomes occluded when covered by multiple devices. ${ }^{8}$ In these situations, competitive collateral flow from the external carotid artery may create a "flow equalization 


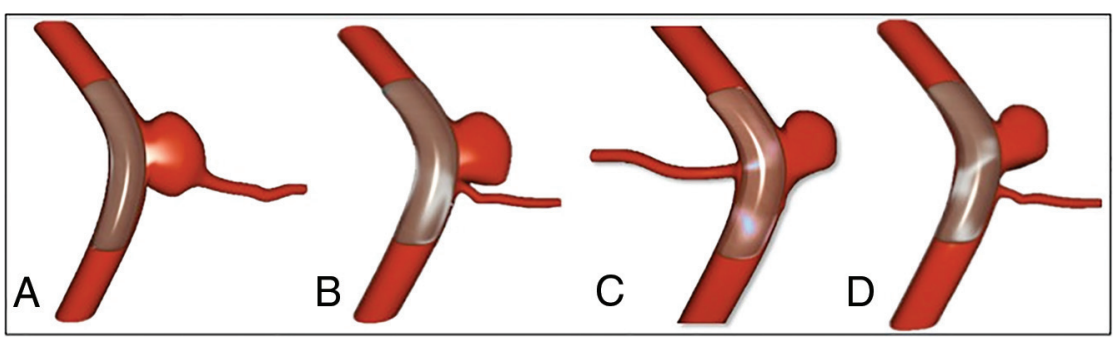
the ophthalmic artery aneurysm classification. $A$, Type $\mathrm{A}$ : ophthalmic artery originating from the aneurysm sac. B, Type B: ophthalmic artery originating from the neck of the aneurysm. C, Type C: ophthalmic artery originating in the inner curve of the carotid siphon. D, Type D: ophthalmic artery not involved in the aneurysm but covered by the Pipeline Embolization Device.
FIG 1. Classification according to the anatomy of the origin of the covered ophthalmic artery:

The number of PEDs to be deployed was decided on the basis of flow reduction observed during the procedure or of anatomic circumstances. Additional coiling was performed in large aneurysms with a maximum diameter of $>12 \mathrm{~mm}$, by using the jailed microcatheter technique.

\section{Periprocedural Medication}

All patients were given dual antiplatelet therapy for 7 days before to 3 months after the intervention (clopidogrel, 75 mg; aspirin, $160 \mathrm{mg}$ daily). Adequate platelet inhibition was verified the day

point," resulting in slow flow or proximal occlusion of the ophthalmic artery because of its retrograde supply from the external carotid artery circulation. ${ }^{15,16}$ Recently, a study evaluated the patency rate of the ophthalmic artery with standard digital subtraction angiography and any change in angiographic flow in the artery immediately after FDS placement across the arterial inlet for the treatment of 20 proximal ICA aneurysms. ${ }^{17}$ This study assessed $21 \%$ of ophthalmic artery occlusions and $11 \%$ of ophthalmic arteries with slow antegrade flow. In this small, retrospective series, no visual worsening was observed in case of flow change in the ophthalmic artery, but only 5 patients had a detailed ophthalmic examination. ${ }^{17}$ Because small visual field amputations due to ischemic lesions can be easily underdiagnosed and they are often asymptomatic, it appears mandatory to explore ophthalmic disorders with a complete examination to identify even small deficits.

In this study, we analyzed ophthalmic consequences due to coverage of the ophthalmic artery by the implantation of an FDS covering the origin of the ophthalmic artery for the treatment of terminal ICA aneurysms. This analysis focuses on the anatomy of the origin of the ophthalmic artery in relation to the target aneurysm.

\section{MATERIALS AND METHODS \\ Patients}

From April 2009 to April 2013, the clinical and angiographic outcomes of all patients treated at our institution for aneurysms with PEDs covering the origin of the ophthalmic artery were collected prospectively. Our institutional ethics committee approved the study. Written informed consent was obtained from each patient.

\section{Procedures}

All procedures were performed by an experienced neuroradiologist with the patient under general anesthesia, by using a triaxial approach. Accurate measurement of the parent artery was performed on 3D images, acquired with rotational angiography, with a dedicated workstation (XtraVision; Philips Healthcare, Best, the Netherlands) for vessel analysis before each procedure. The PEDs (Covidien) were deployed in the desired position to have a perfect wall apposition. Angiographic images were acquired in anteroposterior, lateral, and working projections before and immediately after treatment. To assess the wall positioning of the device, we performed a vaso-CT acquisition (Allura Xper FD20; Philips Healthcare) just after the deployment. ${ }^{18}$ In case of malpositioning, balloon remodeling was performed inside the device or a second overlaid PED was implanted. before treatment with the VerifyNow P2Y12 assay (Accumetrics, San Diego, California).

If there was resistance, the doses were increased.

During catheterization and PED placement, anticoagulation with heparin kept the activated clotting time at 2-3 times the normal value. In addition, $250 \mathrm{mg}$ of aspirin was given intravenously just before PED deployment.

\section{Imaging}

MR imaging was performed to assess optic nerve compression and cerebral ischemic complications with a 3T system before and 1 day and 3 months after the procedure.

\section{Data Collection and Image Analysis}

Patient age, sex, aneurysm location and type (saccular or fusiform), the presence of previous treatment, size, rupture status at presentation, and compressive symptoms were collected. Procedural details (eg, number and size of implanted PEDs and associated coiling) were noted.

A single reader evaluated all angiograms. Aneurysm occlusion was assessed by using the O'Kelly-Marotta grading scale. The patency of the ophthalmic artery was reported as normal or slowed anterograde flow, occlusion, or retrograde filling.

\section{Classification according to Anatomy of the Origin of the Covered Ophthalmic Artery}

The origin of the ophthalmic artery correlated to the location of the aneurysm was assessed by using a 4-type ophthalmic artery aneurysm $\left(\mathrm{OA}^{2}\right)$ classification as detailed in Fig 1.

\section{Ophthalmologic Examination}

A complete standardized ophthalmologic examination was performed by a single ophthalmologist 48 hours before and 1 week after covering the ophthalmic artery. At every visit, possible visual side effects of treatment (decrease of visual acuity, loss of visual field, diplopia, and ocular pain) were investigated.

A routine logarithm of the minimum angle of resolution best spectacle-corrected visual acuity assessment was performed, followed by a slit-lamp examination of the anterior segment, fluorescein staining, a dilated fundus examination, and applanation tonometry. The oculocephalic reflex and a Lancaster test searched the limitation of the extrinsic ocular motility.

Each patient underwent retinal photography and, in case of any abnormality, retinal fluorescein angiography to study retinal 
Table 1: Demographics and aneurysm/procedure characteristics ${ }^{\mathrm{a}}$

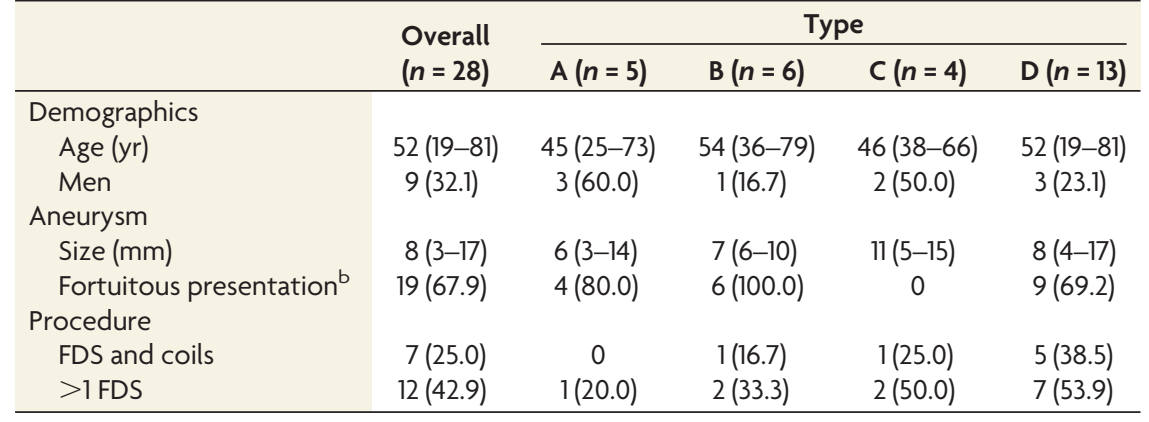

${ }^{a}$ Values reported are median (range) or count (percentage).

b Other cases were recanalization or oculomotor palsy.

Table 2: Outcomes ${ }^{\mathrm{a}}$

\begin{tabular}{|c|c|c|c|c|c|}
\hline & \multirow{2}{*}{$\begin{array}{l}\text { Overall } \\
(n=28)\end{array}$} & \multicolumn{4}{|c|}{ Type } \\
\hline & & $\mathrm{A}(n=5)$ & $\mathrm{B}(n=6)$ & $C(n=4)$ & $D(n=13)$ \\
\hline \multicolumn{6}{|l|}{ Normal patency of ophthalmic artery ${ }^{\mathrm{b}}$} \\
\hline Immediately after procedure & $24(85.7)$ & $5(100)$ & $5(83.3)$ & $2(50.0)$ & $12(92.3)$ \\
\hline At $3 \mathrm{mo}$ & $27(96.4)$ & $5(100)$ & $6(100)$ & $4(100)$ & $12(92.3)$ \\
\hline At $12 \mathrm{mo}$ & $27(96.4)$ & $5(100)$ & $6(100)$ & $4(100)$ & $12(92.3)$ \\
\hline \multicolumn{6}{|l|}{ Ophthalmic complications } \\
\hline Retinal emboli & $5(17.9)$ & $4(80.0)$ & 0 & 0 & $1(7.7)$ \\
\hline Optic atrophy & $3(10.7)$ & 0 & 0 & $3(75.0)$ & 0 \\
\hline New clinical symptoms & $11(39.3)$ & $4(80.0)$ & $1(16.7)$ & $3(75.0)$ & $3(23.1)$ \\
\hline Transient & $6(21.4)$ & $1(20.0)$ & $1(16.7)$ & $1(25.0)$ & $3(23.1)$ \\
\hline Permanent & $5(17.9)$ & $3(60.0)$ & 0 & $2(50.0)$ & 0 \\
\hline
\end{tabular}

${ }^{a}$ Values reported are count (percentage)

${ }^{\mathrm{b}}$ Other cases had occlusion or slowed blood flow. side effects. A Topcon 50 IA camera (Topcon, Tokyo, Japan) coupled with the IMAGEnet Lite (Topcon) digital imaging system was used to acquire the images. Mean retinal thickness in the central foveal area was measured by using optical coherence tomography (Stratus OCT, Version 4.0.1; Carl Zeiss Meditec, Dublin, California). To study the retinal nerve fiber layer, we scanned the optical discs of each patient by optical coherence tomography. For visual field assessment, the 24-2 central field SITA Fast program of the Humphrey 750 (Carl Zeiss Meditec) was performed.

\section{Clinical Events}

Any clinical events appearing in the postoperative course were noted. A neurologic assessment was performed before the treatment, at discharge, and at follow-up.

\section{Angiographic Follow-Up}

Angiographic images obtained immediately after endovascular treatment were compared with those obtained at each follow-up examination. Our standard angiographic follow-up protocol consisted of angiography 3-6 months after endovascular treatment and then 1 year later, by using standard projections and the working projection for PED placement.

\section{Data Analysis}

Continuous variables are expressed as median (range), and qualitative variables, as count (percentage). Baseline characteristics and outcomes are described according to the $\mathrm{OA}^{2}$ classification without statistical comparisons regarding the study sample size. Data were analyzed with SAS, Version 9.3 (SAS Institute, Cary, North Carolina).

\section{RESULTS}

\section{Patients}

Twenty-eight internal carotid artery aneurysms in 28 patients (median age, 52 years; $32.1 \%$ men) were selected for treatment with the FDS covering the origin of the ophthalmic artery (Table 1). Median aneurysm size was $8 \mathrm{~mm}$ (range, 3-17 mm). Presentations were fortuitous in 67.9\%, angiographic recurrence of previously endovascularly treated aneurysms in $17.9 \%$, and acute oculomotor nerve palsy due to nerve compression by the aneurysm in $14.2 \%$.

Aneurysms were treated with an FDS alone in $75 \%$ and with an FDS and coils in $25 \%$ (Table 1). In most cases $(57.1 \%$ ), only 1 FDS was implanted, $35.7 \%$ had 2, and $7.1 \%$ with giant aneurysms had 3 FDSs.

Anatomic configuration according to the origin of the covered ophthalmic artery was type $\mathrm{A}$ in $17.9 \%, \mathrm{~B}$ in $21.4 \%, \mathrm{C}$ in $14.3 \%$, and D in $46.4 \%$, with no important differences among the 4 groups in terms of demographics, aneurysm characteristics, or adjunctive use of coils.

\section{Outcomes}

During endovascular treatment, 1 parenchymal embolism occurred in the territory of the right middle cerebral artery, which was treated with intra-arterial abciximab without any clinical symptoms at patient wake-up, as previously reported. ${ }^{19}$

Follow-up MR imaging, performed in all patients, showed 1 case of territorial parenchymal ischemia in the right frontal area without clinical symptoms and no case of hemorrhagic complications. There were no cases of oculomotor nerve compression due to aneurysm thrombosis, and no patients had optic nerve compression or enlargement of the aneurysm after FDS implantation.

At follow-up, there was a global aneurysm complete occlusion rate of $78.6 \%$ at 6 months, with no difference according to the $\mathrm{OA}^{2}$ classification. No cases of stent thrombosis were observed, and there was no intrastent intimal hyperplasia on DSA. No patients were lost to follow-up.

Ophthalmic artery patency was normal at the end of endovascular treatment in $85.7 \%$ of patients (Table 2). We observed 1 case of ophthalmic artery occlusion in a type B anatomy just after implantation of 2 FDSs with no ophthalmic symptoms despite an exhaustive examination and with a normal antegrade flow at 3-month and 1-year follow-ups. We observed 3 cases of ophthalmic artery flow slowing just after FDS implantation. At 3-month and 1-year follow-ups, all except 1 case had a normal antegrade flow in the covered ophthalmic artery. The only case that was not angiographically normal was a stenosis at the origin of a type D ophthalmic artery.

With an extensive ophthalmic examination, 11 patients 

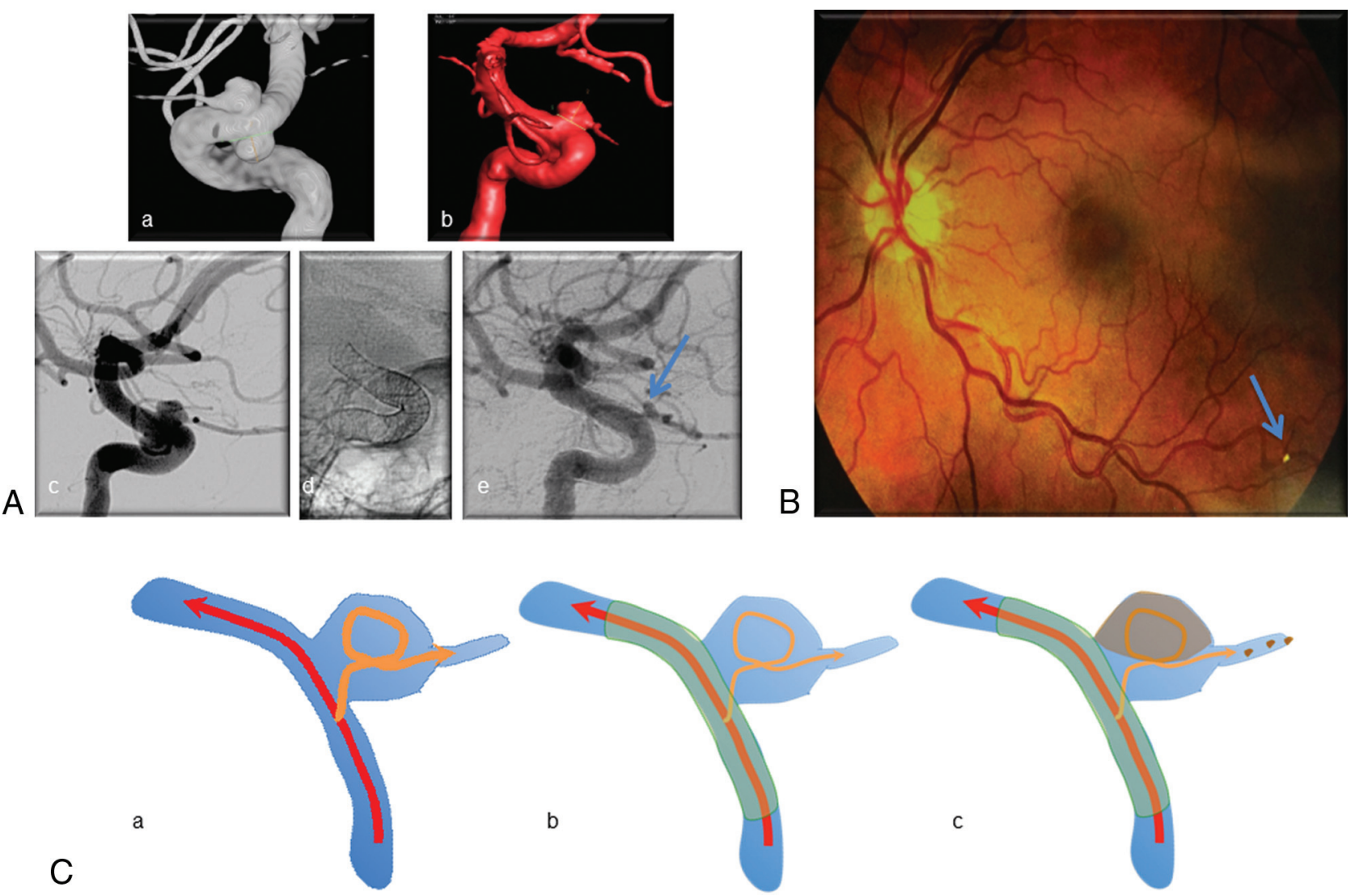

FIG 2. Illustrative case for type A. A, Left internal carotid artery angiograms: $a$ and $b, 3 D$ angiograms with 2 carotid ophthalmic aneurysms with the ophthalmic artery originating from the aneurysm. $c-e$, Angiogram 3 months after flow-diverting stent implantation showing patency of the ophthalmic artery with a remnant of the upper aneurysm (arrow in $e$ ). $B$, Left eye funduscopic examination showing hyperattenuated embolic material in a retinal artery of the inferior retina (arrow). C. Explicative schema: $a$, The flow in the ophthalmic artery is turbulent due to the aneurysm. $b$, After placement of an FDS across the aneurysm neck, the turbulent flow is modified and may induce partial thrombosis of the aneurysm. $c$, The outflow channel that constitutes the ophthalmic artery may be the route for ophthalmic thromboemboli and subsequent retinal infarcts.

(39.3\%) showed new ophthalmic complications: Six had visual blurring, 4 had visual field defects, and 1 had oculomotor palsy. New clinical complications were transient or asymptomatic in 6 ( 2 cases of visual field amputations were asymptomatic but were observed on visual field examinations). We observed 5 (17.9\%) retinal emboli, all but 1 in type $\mathrm{A}$ anatomy, and 3 optic nerve atrophies in a type $\mathrm{C}$ anatomy (Table 2 ).

\section{DISCUSSION}

FDSs offer new opportunities for the treatment of aneurysms, extending the field of aneurysms eligible for endovascular approach and increasing long-term occlusion rates. ${ }^{1,4,15,20}$ They are considered effective and safe, especially before the level of the circle of Willis. Our findings support this finding because we observed only 1 case of parenchymal ischemic complication, no clinical symptoms, and $78.6 \%$ achieving complete occlusion at 6-month follow-up. However, the complications of FDSs are not well-known, and it seems important to detect them to understand their mechanisms and prevent them.

Our results show that coverage of the ophthalmic artery is not a harmless treatment because $17.9 \%$ of patients had permanent ophthalmic complications. These complications can be mild or severe, but some are only detected with a complete and extensive ophthalmic examination, which was not performed in most previous studies.

Puffer et $\mathrm{al}^{17}$ reported that FDSs over the ophthalmic artery were clinically well-tolerated, because no patient experienced visual loss, even though $25 \%$ of the ophthalmic arteries covered with an FDS underwent ophthalmic artery occlusion with time. Some patients may have no clinical symptoms because of asymptomatic loss of the FOV, which highlights the importance of extensive ophthalmic examinations to detect retinal ischemia or subtle deficits.

The arterial flow of the ophthalmic artery, analyzed with DSA, may not be the only predictor of ophthalmic complications after coverage of the ophthalmic artery, especially because asymptomatic delayed ophthalmic artery thrombosis may be due to the development of an important collateral network. ${ }^{17,21}$ This mechanism is supported by several animal studies, ${ }^{8,22}$ suggesting that in cases of laminar flow with aspiration effect, due to a high pressure gradient in the covered side branch, the ophthalmic artery remains patent, but if the pressure gradient is low, as happens with the ophthalmic artery where an important collateral network develops, flow diversion may be increased and can result in side branch thrombosis. The aspirative flow mechanism to explain the patency of covered arteries has been described in a rabbit model 

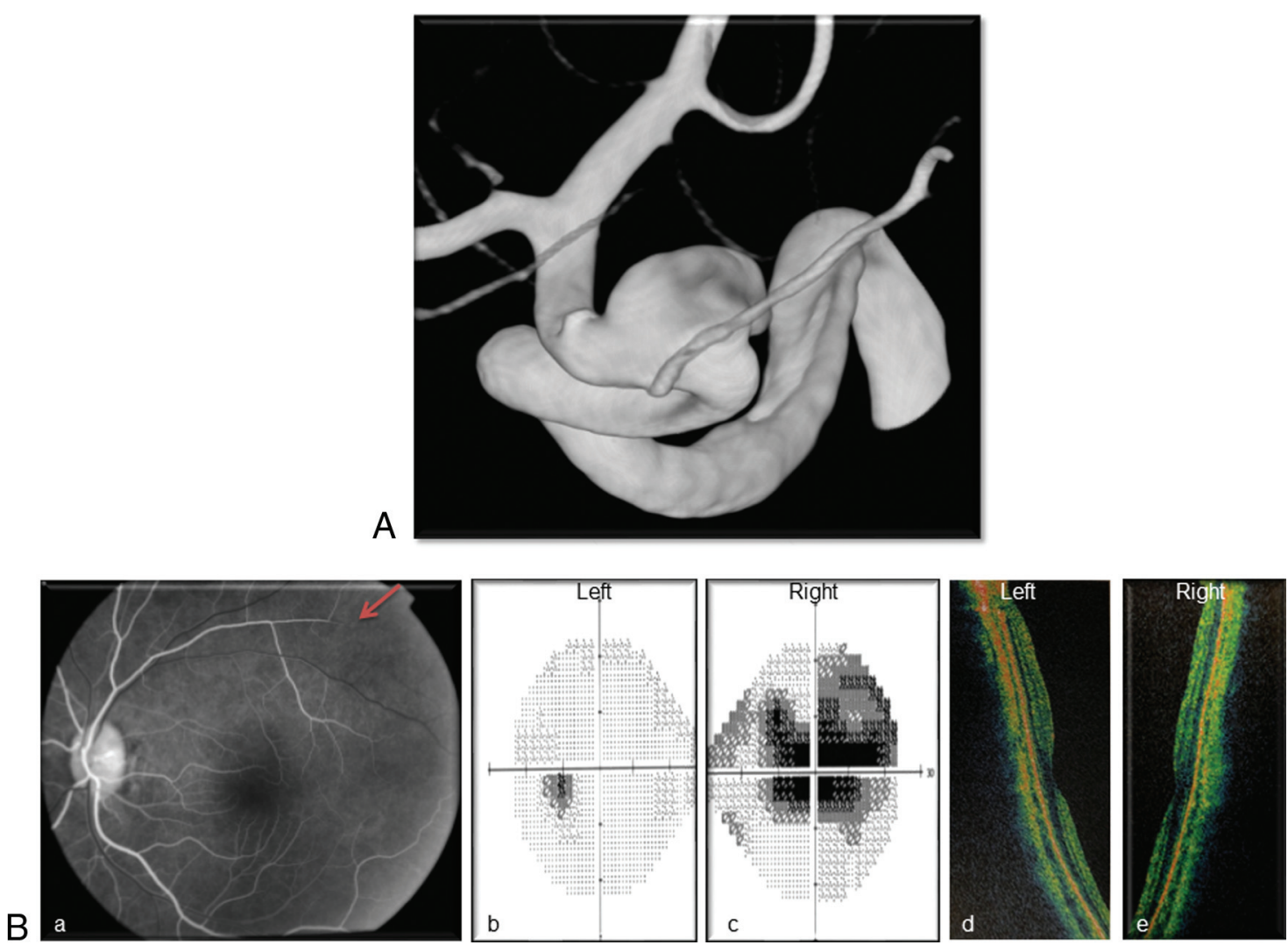

FIG 3. Illustrative case of type $C$. $A$, Right internal carotid artery $3 D$ angiogram showing a carotid ophthalmic aneurysm with the ophthalmic artery originating from the inner curve of the ICA. $B$, Three-month ophthalmic examination: $a$, Right eye fluorescein retinal angiography showing an arterial flow defect in the upper part of the retina (arrow). $b$ and $c$, Visual field assessment showing a large central scotoma in the right eye. $d$ and $e$, Mean retinal thickness in the central foveal area, measured by using optical coherence tomography, proved right optic nerve atrophy (right eye, $55.03 \mu \mathrm{m}$; left eye, $100.65 \mu \mathrm{m}$ ).

with coverage of the lumbar arteries; the authors reported excellent patency of small branch arteries covered by the device. ${ }^{15}$ This hypothesis, which has been confirmed in clinical practice, is that continued flow in these arteries permits ongoing patency, whereas aneurysm cavities, which lack an outflow channel, undergo thrombosis due to blood stagnation. ${ }^{8,22}$ It may be that this mechanism is not exactly applicable to all anatomic configurations; we therefore classified aneurysms into 4 groups according to the $\mathrm{OA}^{2}$ classification (Fig 1).

In the case of an aneurysm at the origin of the covered side branches with the ophthalmic artery originating from the aneurysm sac (Fig 1A), the ophthalmic artery has turbulent flow after crossing the aneurysm rather than a rapid laminar flow. Thus, after placement of an FDS across the aneurysm neck, the turbulent flow in the aneurysm is modified and may induce partial thrombosis. Furthermore, the outflow channel that constitutes the ophthalmic artery may be the route for ophthalmic thromboemboli and subsequent retinal infarcts. Despite the small number of patients with type A aneurysms in our study $(n=5)$, we observed small retinal emboli in $80.0 \%$ of these cases. The hypothetic mechanism for this is illustrated in Fig 2. It may be that for these particular cases of a branched artery originating from the aneurysm sac, it would be necessary to restore a laminar flow in the branch with coiling before implantation of the FDS, but this strategy should be tested in animal models or with fluid computational analysis.
Another observation of this study is that patients with an ophthalmic artery originating in the inner curve of the carotid siphon (Fig 1C) have high rates of complications. This configuration only accounted for $14.3 \%$ of patients in our series, but $75.0 \%$ of them had new ophthalmic complications. Three of the 4 patients with type $\mathrm{C}$ anatomy presented with optic nerve atrophy, as described in Fig 3. The hypothesis to explain optic nerve atrophy is ischemia due to a drastic and rapid reduction of the blood flow in the ophthalmic artery. This could be explained by the tortuosity of the carotid siphon and the braided design of the FDS. In the inner part of a curve, the mesh attenuation of the device is higher than that in the outer curve because of tighter packing of the FDS. ${ }^{23,24}$ The free-cell space is consequently reduced across the origin of the ophthalmic artery, which leads to a rapid decrease of the inflow of the vessel. ${ }^{23,24}$ The persistency of an aspirative effect may allow patency of the ophthalmic artery, but it induces a defect of perfusion. If antegrade inflow by the ophthalmic artery decreases, its territory is taken over by the collaterals from the external carotid artery with a competitive flow through anastomosis, but this network develops with time.

In case of acute low perfusion and before any supply by collateral branches from the external carotid system, some territories vascularized by very tiny branches can be hypoperfused. This hypoperfusion might occur for the optic nerve head perfusion, which is ensured by perforators from short posterior ciliary arteries constituting the Zinn-Haller arterial ring. ${ }^{25-27}$ These territo- 
ries can be injured in case of low flow after resuscitation or surgery and can cause optic nerve ischemia. ${ }^{28,29}$ This mechanism of acute low flow in the ophthalmic artery after implantation of an FDS with high mesh attenuation over the ophthalmic artery might explain optic nerve ischemia despite normal angiography findings. A type $\mathrm{C}$ aneurysm must be a concern before FDS implantation, and it appears mandatory to strictly adapt the FDS diameter to that of the carotid artery and choose an FDS of larger diameter to avoid excessive packing and reduce mesh attenuation in the inner curve.

Our study is limited by the small number of patients with each $\mathrm{OA}^{2}$ classification, which lowers its power to identify differences among each type, and the study sample size is not large enough to exclude the possibility of confounding factors. However, the data of this series identify a large tendency for more ophthalmic complications (permanent or definitive) in groups $\mathrm{A}$ and $\mathrm{C}$ compared with other anatomy configurations. We did not observe an increased risk of ophthalmic complications related to the number of overlaid PEDs or in cases of associated coiling (data not shown), suggesting a real relationship between ophthalmic artery anatomy and ophthalmologic outcome, which should be confirmed in a larger population.

Furthermore, this observational study had no control group, and we did not perform detailed ophthalmic investigations in carotid-ophthalmic aneurysms treated with other devices or in the absence of coverage of the ophthalmic artery with the FDS. Nevertheless, the series of D'Urso et $\mathrm{al}^{30}$ reported only 1 case of visual impairment in 126 unruptured paraclinoid aneurysms treated with coiling, suggesting that coiling (with or without associated stent) does not carry a high risk of ophthalmic complications and that the observations reported in our series could be related to coverage of the ophthalmic artery by the FDS.

Finally, the patency and flow velocity in the ophthalmic artery were estimated with angiography, which is not the most suitable tool. Further studies should analyze this flow by using Doppler sonography, before and after FDS placement, to correlate ophthalmic complications with subtle ophthalmic artery flow variations.

\section{CONCLUSIONS}

This prospective study shows that covering the ophthalmic artery with an FDS is not without potential complications. Ophthalmic complications are not rare but are usually underdiagnosed. The anatomic disposition of the ophthalmic artery regarding the carotid siphon and aneurysms should be clearly understood because some particular configurations may expose patients to a higher risk. When not required, covering the ophthalmic artery with an FDS should be avoided.

\section{REFERENCES}

1. Brinjikji W, Murad MH, Lanzino G, et al. Endovascular treatment of intracranial aneurysms with flow diverters: a meta-analysis. Stroke 2013;44:442-47

2. Fiorella D, Woo HH, Albuquerque FC, et al. Definitive reconstruction of circumferential, fusiform intracranial aneurysms with the Pipeline embolization device. Neurosurgery 2008;62:1115-20; discussion 1120-21

3. Pistocchi S, Blanc R, Bartolini B, et al. Flow diverters at and beyond the level of the circle of Willis for the treatment of intracranial aneurysms. Stroke 2012;43:1032-38

4. Lylyk P, Miranda C, Ceratto R, et al. Curative endovascular reconstruction of cerebral aneurysms with the Pipeline embolization device: the Buenos Aires experience. Neurosurgery 2009;64:632-42; discussion 642-43

5. D’Urso PI, Lanzino G, Cloft HJ, et al. Flow diversion for intracranial aneurysms: a review. Stroke 2011;42:2363-68

6. Pierot L. Flow diverter stents in the treatment of intracranial aneurysms: where are we? J Neuroradiol 2011;38:40-46

7. Çinar C, Bozkaya H, Oran I. Endovascular treatment of cranial aneurysms with the Pipeline flow-diverting stent: preliminary midterm results. Diagn Interv Radiol 2013;19:154-64

8. Dai D, Ding YH, Kadirvel R, et al. Patency of branches after coverage with multiple telescoping flow-diverter devices: an in vivo study in rabbits. AJNR Am J Neuroradiol 2012;33:171-74

9. Byrne JV, Beltechi R, Yarnold JA, et al. Early experience in the treatment of intra-cranial aneurysms by endovascular flow diversion: a multicentre prospective study. PloS One 2010;5:e12492

10. Lubicz B, Collignon L, Raphaeli G, et al. Flow-diverter stent for the endovascular treatment of intracranial aneurysms: a prospective study in 29 patients with 34 aneurysms. Stroke 2010;41:2247-53

11. Leonardi M, Cirillo L, Toni F, et al. Treatment of intracranial aneurysms using flow-diverting Silk stents (BALT): a single centre experience. Interv Neuroradiol 2011;17:306-15

12. Lubicz B, Collignon L, Raphaeli G, et al. Pipeline flow-diverter stent for endovascular treatment of intracranial aneurysms: preliminary experience in 20 patients with 27 aneurysms. World Neurosurg 2011;76:114-19

13. Sadasivan C, Cesar L, Seong J, et al. An original flow diversion device for the treatment of intracranial aneurysms: evaluation in the rabbit elastase-induced model. Stroke 2009;40:952-58

14. Kulcsár Z, Ernemann U, Wetzel SG, et al. High-profile flow diverter (Silk) implantation in the basilar artery: efficacy in the treatment of aneurysms and the role of the perforators. Stroke 2010;41:1690-96

15. Fiorella D, Lylyk P, Szikora I, et al. Curative cerebrovascular reconstruction with the Pipeline embolization device: the emergence of definitive endovascular therapy for intracranial aneurysms. J Neurointerv Surg 2009;1:56-65

16. Albuquerque FC. Visual impairment after endovascular treatment: does stent placement increase the incidence? World Neurosurg 2012;78:222

17. Puffer RC, Kallmes DF, Cloft HJ, et al. Patency of the ophthalmic artery after flow diversion treatment of paraclinoid aneurysms. J Neurosurg 2012;116:892-96

18. Clarençon F, Piotin M, Pistocchi S, et al. Evaluation of stent visibility by flat panel detector $\mathrm{CT}$ in patients treated for intracranial aneurysms. Neuroradiology 2012;54:1121-25

19. Mounayer C, Piotin M, Baldi S, et al. Intraarterial administration of abciximab for thromboembolic events occurring during aneurysm coil placement. AJNR Am J Neuroradiol 2003;24:2039-43

20. Becske T, Kallmes DF, Saatci I, et al. Pipeline for uncoilable or failed aneurysms: results from a multicenter clinical trial. Radiology 2013;267:858-68

21. Szikora I, Berentei Z, Kulcsar Z, et al. Treatment of intracranial aneurysms by functional reconstruction of the parent artery: the Budapest experience with the Pipeline embolization device. AJNR Am J Neuroradiol 2010;31:1139-47

22. Kallmes DF, Ding YH, Dai D, et al. A second-generation, endoluminal, flow-disrupting device for treatment of saccular aneurysms. AJNR Am J Neuroradiol 2009;30:1153-58

23. Makoyeva A, Bing F, Darsaut TE, et al. The varying porosity of braided self-expanding stents and flow diverters: an experimental study. AJNR Am J Neuroradiol 2013;34:596-602

24. Maimon S, Gonen L, Nossek E, et al. Treatment of intra-cranial aneurysms with the Silk flow diverter: 2 years' experience with 28 patients at a single center. Acta Neurochir (Wien) 2012;154:979-87 
25. Hayreh SS. The 1994 Von Sallman Lecture: the optic nerve head circulation in health and disease. Exp Eye Res 1995;61:259-72

26. Ohno-Matsui K, Futagami S, Yamashita S, et al. Zinn-Haller arterial ring observed by ICG angiography in high myopia. $\mathrm{Br} J \mathrm{Ophthalmol}$ 1998;82:1357-62

27. Jonas JB, Holbach L, Panda-Jonas S. Peripapillary arterial circle of Zinn-Haller: location and spatial relationships with myopia. PloS One 2013;8:e78867

28. Wirth CD, Leitner C, Perrig M. Bilateral posterior ischaemic optic neuropathy after severe diabetic ketoacidosis, cardiopulmonary resuscitation and respiratory failure. BMJ Case Rep 2013;2013:pii: bcr2012008291

29. Kudo D, Yamamura H, Nishiuchi T, et al. Anterior and posterior ischemic optic neuropathy related to massive fluid resuscitation after blunt trauma. J Trauma 2010;68:E67-70

30. D'Urso PI, Karadeli HH, Kallmes DF, et al. Coiling for paraclinoid aneurysms: time to make way for flow diverters? AJNR Am J Neuroradiol 2012;33:1470-74 